\title{
Flow Reversal in Traveling-Wave Electrokinetics: An Analysis of Forces Due to Ionic Concentration Gradients
}

\author{
P. García-Sánchez* ${ }^{\dagger}$ and A. Ramos \\ Departamento de Electrónica y Electromagnetismo, Facultad de Física, Universidad de Sevilla, \\ 41012, Seville, Spain. ${ }^{\dagger}$ Present address: Physics of Complex Fluids, Faculty of Science \\ and Technology, MESA ${ }^{+}$Institute, University of Twente, PO Box 217, 7500 AE Enschede, \\ The Netherlands.
}

\author{
A. González \\ Departamento de Física Aplicada III, Escuela Superior de Ingenieros, Universidad de Sevilla, 41092, \\ Seville, Spain \\ N. G. Green and H. Morgan \\ School of Electronics and Computer Science, University of Southampton, SO17 1BJ, Southampton, \\ United Kingdom
}

Received November 3, 2008. Revised Manuscript Received February 12, 2009

\begin{abstract}
Pumping of electrolytes using ac electric fields from arrays of microelectrodes is a subject of current research. The behavior of fluids at low signal amplitudes $\left(<2-3 \mathrm{~V}_{\mathrm{pp}}\right)$ is in qualitative agreement with the prediction of the ac electroosmosis theory. At higher voltages, this theory cannot account for the experimental observations. In some cases, net pumping is generated in the direction opposite to that predicted by the theory (flow reversal). In this work, we use fluorescent dyes to study the effect of ionic concentration gradients generated by Faradaic currents. We also evaluate the influence of factors such as the channel height and microelectrode array shape in the pumping of electrolytes with traveling-wave potentials. Induced charge beyond the Debye length is postulated to be responsible for the forces generating the observed flows at higher voltages. Numerical calculations are performed in order to illustrate the mechanisms that might be responsible for generating the flow.
\end{abstract}

\section{Introduction}

Laboratory-on-a-Chip technologies demand the development of new techniques for liquid control on the micrometer scale. ${ }^{1-5}$ Electrodes integrated within microchannels represent an opportunity for direct actuation on either the liquid or the suspended particles. Therefore, electrohydrodynamics (EHD) in microsystems is of central interest in current microfluidics research. Among the multiple possibilities of using electric fields, the realization of micropumps without moving parts is one that has received major attention. ${ }^{3,6}$ Electroosmosis, ${ }^{7}$ electrowetting, ${ }^{8}$ iondrag pumping, ${ }^{9}$ electrohydrodynamic induction pumping, ${ }^{10}$ and ac electroosmosis (ACEO) ${ }^{11}$ are examples of phenomena where electric fields are used for driving fluids within micrometer dimension systems.

*pablogarcia@us.es.

(1) Stone, H.; Stroock, A.; Ajdari, A. Annu. Rev. Fluid Mech. 2004, 36, 381411.

(2) Squires, T. M.; Quake, S. R. Rev. Mod. Phvs. 2005, 77, 977-1026.

(3) Ramos, A. Electrohydrodynamics and Magnetohydrodynamics Micropumps. In Microfluidics technologies for miniaturized analysis systems; Springer, 2008 .

(4) Whitesides, G.; Janasek, D.; Franzke, J.; et al. Nature (London) 2006, 442, $367-418$.

(5) Dittrich, P.; Tachikawa, K.; Manz, A. Anal. Chem. 2006, 2, 3887-3908.

(6) Laser, D. J.; Santiago, J. G. J. Micromech. Microeng. 2004, 14, R35-R64.

(7) Pretorius, V.; Hopkins, B.; Schieke, J. J. Chromatogr. 1974, 99, 23-30.

(8) Beni, G.; Tenan, M. J. Appl. Phvs. 1981, 52, 6011-6015.

(9) Richter, A.; Sandmaier, H. IEEE 1990, 99-104.

(10) Fuhr, G.; Hagedorn, R.; Muller, T.; Benecke, W.; Wagner, B. J. Microelectro-mech. Systems 1992, 1, 141-146.

(11) Brown, A. B. D.; Smith, C. G.; Rennie, A. R. Phys. Rev. E 2000, 63, 016305.
AC electroosmotic ${ }^{12,13}$ pumping is based on the use of arrays of microelectrodes connected to low-voltage ac signals (less than $10 \mathrm{~V})$. The microelectrode arrays are fabricated on glass and mounted in a microfluidic channel. Typically, the channel contains an electrolyte with conductivity ranging between $10^{-4} \mathrm{~S} / \mathrm{m}$ and $10^{-1} \mathrm{~S} / \mathrm{m}$. Net fluid flow is observed after application of an electric potential. Most of the work on ACEO pumping has been carried out with arrays of pairs of asymmetric electrodes with a single-phase ac signal. ${ }^{11,14-17}$ Arrays of electrodes subjected to traveling-wave (TW) potentials is an alternative driving mechanism that can achieve unidirectional fluid flow. ${ }^{18,19}$ Figure 1 shows the basic mechanism of traveling-wave electroosmosis (TWEO) where electrodes are connected to a TW potential. The electric field induces charge at the electrode/electrolyte interface, i.e., the electrical double layer. This charge is pulled in the direction of the traveling wave, and therefore, unidirectional net flow is

(12) Ramos, A.; Morgan, H.; Green, N.; Castellanos, A. J. Colloid Interface Sci. 1999, 217, 420-422.

(13) Green, N.; Ramos, A.; González, A.; Morgan, H.; Castellanos, A. Phys. Rev. E 2000, 61, 4011-4018.

(14) Ajdari, A. Phys. Rev. E 2000, 61, R45-R48.

(15) Studer, V.; Pépin, A.; Chen, Y.; Ajdari, A. The Analyst 2004, 29, 944-949.

(16) Urbanski, J.; Thorsen, T.; Levitan, J.; Bazant, M. Appl. Phys. Lett. 2006, 89,143508 .

(17) Gregersen, M. M.; Olesen, L. H.; Brask, A.; Hansen, M. F.; Bruus, H. Phys. Rev. E 2007, 76, 056305.

(18) Cahill, B. P.; Heyderman, L. J.; Gobrecht, J.; Stemmer, A. Phys. Rev. E 2004, 70, 036305 .

(19) Ramos, A.; Morgan, H.; Green, N. G.; González, A.; Castellanos, A. J. Appl. Phvs. 2005, 97, 084906. 


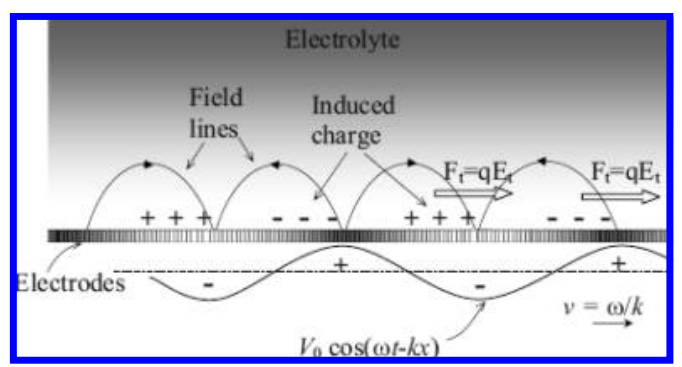

Figure 1. Physical mechanism of traveling-wave electroosmosis. A sinusoidal potential travels to the right. Electrical charge is induced at the electrode/electrolyte interface and a net force pulls the charges.

obtained. Other examples where ACEO flows have been exploited for either pumping or mixing in microsystems can be found in the literature. $^{20-24}$

In experiments, a traveling-wave potential is usually implemented by applying four phase-shifted ac signals to an array of equal-sized electrodes (see Figure 2). Electrolyte pumping with a four-phase TW was demonstrated by Cahill et al. ${ }^{18}$ and Ramos et al. ${ }^{19}$ For sufficiently low amplitude signals $\left(<2-3 \mathrm{~V}_{\mathrm{pp}}\right)$, the ac electroosmosis theory explains the experimental observations of pumping, although a more quantitative agreement is still lacking. The experimental observations at higher voltages cannot be explained by the theory; even the direction of flow is opposite to prediction (from now on, we refer to this as flow reversal). ${ }^{25,26}$

Fluorescent latex beads are usually employed in microfluidic experiments for fluid flow visualization. Figure 3 shows pathlines of these beads over a traveling-wave array of $20 \mu \mathrm{m}$ wide titanium microelectrodes separated by $20 \mu \mathrm{m}$ gaps; the liquid was a solution of $\mathrm{KCl}$ in water at a concentration of $0.1 \mathrm{mM}$. Figure $3 \mathrm{a}$ shows data for an applied signal of $2.4 \mathrm{~V}_{\mathrm{pp}}$ and $1 \mathrm{kHz}$ (reprinted from Ramos et al. ${ }^{19}$ ). In this case, the net flow is in the direction predicted by the ac electroosmosis theory and the pathlines fit to the theoretical streamlines expected by theory. Figure $3 \mathrm{~b}$ is for an applied signal of $4.13 \mathrm{~V}_{\mathrm{pp}}$ and $800 \mathrm{~Hz}$. The array is pumping the liquid in the reverse direction. No theory currently predicts the shape of these pathlines, but they are clearly different from the pathlines at low voltage. These pathlines cannot be obtained just by changing the velocity direction at the level of the electrodes. Fluorescent beads accumulate at the center of the electrodes, and some beads are strongly ejected in the vertical direction, forming clockwise-rotating "flow rolls" on the right side of the electrodes (the potential travels from right to left). Particles are also subjected to electrical forces near the electrodes; these particles do not accurately map the fluid flow.

Flow reversal has also been found in experiments with arrays of asymmetric pairs of electrodes. For instance, in Studer et al. ${ }^{15}$ the fluid flow was reversed at higher voltages, but for frequencies higher than $20 \mathrm{kHz}$ (much greater than in the case of TWEO). Recent experiments with asymmetric arrays ${ }^{17}$ have shown flow reversal at low voltage and frequencies, but only for ionic concentrations equal to or greater than $400 \mu \mathrm{M}$. The different

(20) Lastochkin, D.; Zhou, R.; Wang, P.; Ben, Y.; Chang, H. J. Appl. Phvs. 2004, 96, 1730-1733.

(21) Gagnon, Z.; Chang, H. Electrophoresis 2005, 26, 3725-3737.

(22) Wang, S; Chen, H.; Lee, C.; Yu, C.; Chang, H. Biosens. Bioelectron. 2006, $22,563-567$.

(23) Wang, S; Chen, H.; Chang, H. Biomicrofluidics 2007, 1, 034106.

(24) Harnett, C. K.; Templeton, J.; Dunphy-Guzman, K. A.; Senousy, Y. M.; Kanouff, M. P. Lab Chip 2008, 8, 565-572.

(25) García-Sánchez, P.; Ramos, A.; Green, N. G.; Morgan, H. IEEE Trans. Dielectr. Electr. Insulat. 2006, 13, 670-677.

(26) García-Sánchez, P.; Ramos, A.; Green, N. G.; Morgan, H. Langmuir 2008, $24,9361-9369$

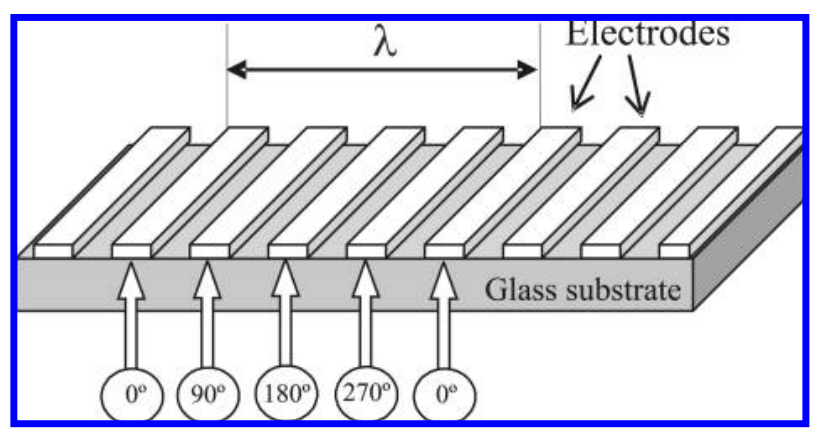

Figure 2. Microelectrode array energized with four phase-shifted voltage sources.

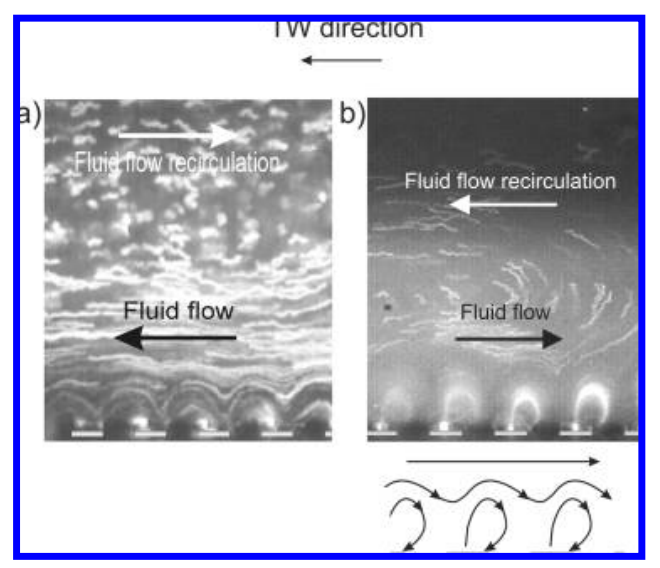

Figure 3. Bead pathlines over a traveling-wave array. The electrodes are $20 \mu \mathrm{m}$ wide and made of titanium. (a) TW signal of $2.4 \mathrm{~V}_{\mathrm{pp}}$ and $1 \mathrm{kHz}$ (normal flow). (b) TW signal of $4.13 \mathrm{~V}_{\mathrm{pp}}$ and $800 \mathrm{~Hz}$ (reverse flow); the drawing below shows the particle motion direction. Typical values for the velocity in these experiments are on the order of $100 \mu \mathrm{m} / \mathrm{s}$.

observations may therefore have different physical origins. It seems that the flow always reverses for specific values of amplitude and frequency. Much theoretical effort is currently devoted to finding an explanation for these phenomena. Theories for generalizing the ACEO model have evolved in two distinct directions: (1) inclusion of Faradaic currents into the standard electrokinetic model ${ }^{27,28}$ and (2) modification of the PoissonBoltzmann equation to account for the finite size of the ions ${ }^{29,30}$ (steric effects must limit the maximum packing of ions). The models that include Faradaic currents are linear, or weakly nonlinear, theories that represent a more general approach to the polarization of the electrode/electrolyte interface at low applied voltages - electrochemical reactions can occur. However, they are not successful in predicting the observed flow reversal. On the other hand, inclusion of steric effects due to the finite size of ions has yielded a possible explanation for flow reversal ${ }^{31}$ in asymmetric arrays, although unrealistic ion sizes are required for a quantitative agreement with experiments. This prediction is only valid for arrays of electrodes of different widths and for frequencies higher than for the reversal observed with travelingwave potentials (where all electrodes are the same size). However, the flow reversal does not necessarily have the same origin in both cases.

(27) Olesen, L; Bruus, H.; Ajdari, A. Phys, Rev. E 2006, 73, 056313.

(28) Ramos, A.; González, A.; García-Sánchez, P.; Castellanos, A. J. Colloid Interface Sci. 2007, 309, 323-331.

(29) Kilic, M. S.; Bazant, M. Z.; Ajdari, A. Phys. Rev. E 2007, 75, 021502.

(30) Kilic, M. S.; Bazant, M. Z.; Ajdari, A. Phys. Rev. E 2007, 75, 021503.

(31) Storey, B.; Kilic, M.; Bazant, M. Phys. Rev. E 2008, 77, 036317. 
In a previous paper, ${ }^{26}$ we reported experiments with arrays of platinum electrodes subjected to traveling-wave potentials. Measurements of the impedance of the system indicated changes in the electrical properties of the electrolyte. The magnitude of the electrical current during pumping of the electrolyte was also measured, and transient behavior in both current and fluid velocity was observed and correlated. In that work, we suggested that Faradaic currents were generating ionic concentration gradients in the liquid bulk, and therefore, electrical charge could be induced beyond the diffuse layer. In the present work, additional experimental evidence is presented as follows: (1) Fluorescent dyes are used to obtain information on the behavior of the electrolyte at ac signals typical of the reported pumping. (2) We check for the influence of electrode array shape and channel height on the flow reversal. The results of these experiments lead us to confirm that there should be induced charge beyond the diffuse layer and this charge could generate a net flow over the electrode array.

Finally, numerical calculations are performed with the aim of illustrating the different mechanisms proposed for the generation of net flow and, particularly, if they lead to flow reversal. The net velocity is calculated for typical experimental parameters and the relative importance of each mechanism is discussed from the standpoint of these calculations.

\section{Experimental Results}

2.1. Fluorescent Dyes. Fluorescent dyes were used to investigate the electrolyte behavior: Rhodamine 6G (SigmaAldrich), Bodipy 492/515 disulfonate (Invitrogen), and Fluorescein (Riedel de Häen). Rhodamine 6G disassociates completely in water to give a single positively charged ion. Bodipy is divalent and dissociates completely to give a negatively charged molecule in water with two charges. Fluorescein is weakly acidic: the molecule partially dissolves in water, giving a negatively charged molecule and a free proton. The equilibrium constant between the dissociated and nondissociated forms of the molecule is $\mathrm{p} K_{\mathrm{a}}=6.4$. Because the dissociated form is fluorescent, changing the $\mathrm{pH}$ changes the degree of ionization of the molecule and therefore the fluorescence intensity.

Fluorescein $^{32}$ has been used for detecting and measuring $\mathrm{pH}$ gradients created by electrochemical reactions over microelectrode arrays. Bodipy and Rhodamine $6 \mathrm{G}^{33}$ have been used as ion tracers for determining the polarization of the ionic concentration in porous glass beads. Bodipy is an anionic tracer, and by monitoring changes in the fluorescence profile, changes in the concentration of negative ions can be deduced. Rhodamine $6 \mathrm{G}$ plays the same role but for positive ions. The fluorescence profile was measured at different electric field strengths - the concentration fields were then estimated. In another publication, ${ }^{34}$ the two dyes were used for tracing ionic concentrations, and the observed fluid motion was described as induced charge electroosmosis. Bodipy and Rhodamine $6 \mathrm{G}$ have also been used to trace electrolyte concentration over floating electrodes within a microchannel. ${ }^{35}$

Dyes were prepared at a concentration of $10^{-5} \mathrm{M}$, maintaining the ion concentration at least 1 order of magnitude below the ionic strength of the electrolyte $\left(\mathrm{KCl} 10^{-4} \mathrm{M}\right)$. The electrolyte conductivity is $1.5 \mathrm{mS} / \mathrm{m}$ and did not noticeably change after the addition of the fluorescent dyes.

We first analyzed the behavior of the fluorescent dyes in a simple geometry: two parallel planar electrodes (Figure 4). Two

(32) Fiedler, S.; Hagedorn, R.; Schnelle, T.; Richter, E.; Wagner, B.; Fuhr, G. Anal. Chem. 1995, 67, 820-828.

(33) Leinweber, F. C.; Tallarek, U. Langmuir 2004, 20, 11637-11648.

(34) Leinweber, F. C.; Tallarek, U. J. Phys. Chem. B 2005, 109, 21481-21485.

(35) Leinweber, F. C.; Eijkel, J. C. T.; Bomer, J. G.; van den Berg, A. Anal. Chem. 2006, 78, 1425-1434.

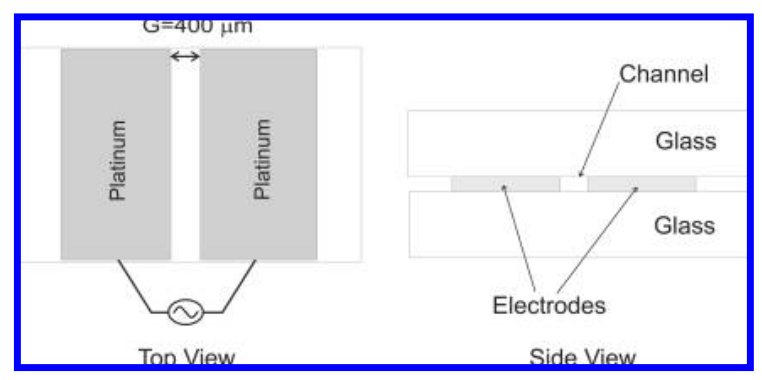

Figure 4. Platinum electrodes in a simple geometry for the study of the fluorescent dyes. The channel is filled with electrolyte containing the dyes. The fluorescence emission can be observed from the top.

platinum plates were placed as shown in the figure. The plates are $100 \mu \mathrm{m}$ thick, with a separation between the two of $G=400 \mu \mathrm{m}$. The plates are stuck to glass slides with double-sided sticky tape. The gap between the plates was filled with electrolyte $\left(\mathrm{KCl} 10^{-4} \mathrm{M}\right)$ containing one of the fluorescent dyes. The device was placed under the microscope objective and connected to a signal generator.

Prior to the application of a signal, a homogeneous fluorescence intensity was observed. When a dc voltage of a few volts was applied, the fluorescence intensity near both electrodes changed. When Bodipy and Rhodamine were used, the electrolyte near the positive electrode became brighter, while the electrolyte near the other electrode became darker than the average brightness. On the other hand, Fluorescein was brighter near the electrode at a lower potential. For ac voltages, the fluorescence intensity oscillated from one electrode to the other at the same frequency as the applied voltage. Bodipy and Rhodamine were brighter near the positive electrode, while Fluorescein became darker near that electrode. This behavior for all three fluorescent dyes was confirmed for both dc and ac signals at frequencies low enough to allow the fluorescence intensity oscillations to be followed by eye $(f \leq 10 \mathrm{~Hz})$. Rhodamine $6 \mathrm{G}$ and Bodipy map concentrations of positive and negative ions, respectively. The two dyes oscillate in phase, indicating changes in the concentration of neutral salt (concentration polarization).

Figure 5 shows the fluorescence emission from an electrolyte containing Bodipy at a certain instant when the pair of electrodes was subjected to an ac potential of $6 \mathrm{~V}_{\mathrm{pp}}$ at a frequency of $0.5 \mathrm{~Hz}$. The electrode in the figure was negative, and a minimum of fluorescence intensity is observed near that electrode.The intensity profile for a given video-frame was obtained with the aid of ImageJ. ${ }^{36}$ From the image sequence, a typical penetration length for the fluorescence oscillations was measured. This distance can be considered a measurement of the diffusion length, assuming that the fluorescent dye is a measure of the ion concentrations. Measurements were repeated for different frequencies, and the data are shown in Figure 6. The experiment was also carried out for a signal amplitude of $10 \mathrm{~V}_{\mathrm{pp}}$, giving the same measurements of diffusion length. The diffusion length $\delta$ is expected to be proportional to the square root of the diffusion coefficient and decrease with the square root of frequency $\delta=(D / \omega)^{1 / 2}$. The experimental data were fitted to a power law model $\delta=A \cdot f^{p}$. The fitting parameter $p$ is $-0.56 \pm$ 0.04 , which is close to the expected theoretical value of -0.5 . The diffusion coefficient can be calculated from the parameter $A$, giving $D=1.36 \times 10^{-9} \mathrm{~m}^{2} / \mathrm{s}$, which is typical for the diffusion coefficient of ionic species, ranging between ${ }^{37} 0.6 \times 10^{-9}$ and $2 \times 10^{-9} \mathrm{~m}^{2} / \mathrm{s}$.

Lower voltages were required to allow observation of changes in the fluorescence emission of the Fluorescein compared with

(36) ImageJ - http://rsb.info.nih.gov/ij/.

(37) Newman, J. S.; Thomas-Alyea, K. E. Electrochemical systems; WileyIEEE, 2004 


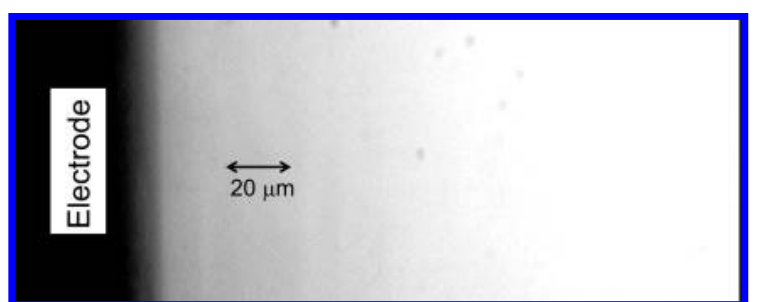

Figure 5. Fluorescence intensity of Bodipy for an applied signal of $6 \mathrm{~V}_{\mathrm{pp}}$ and $0.5 \mathrm{~Hz}$. The electrode in the figure is negative. The decrement in fluorescence intensity indicates a lower concentration of Bodipy near that electrode.

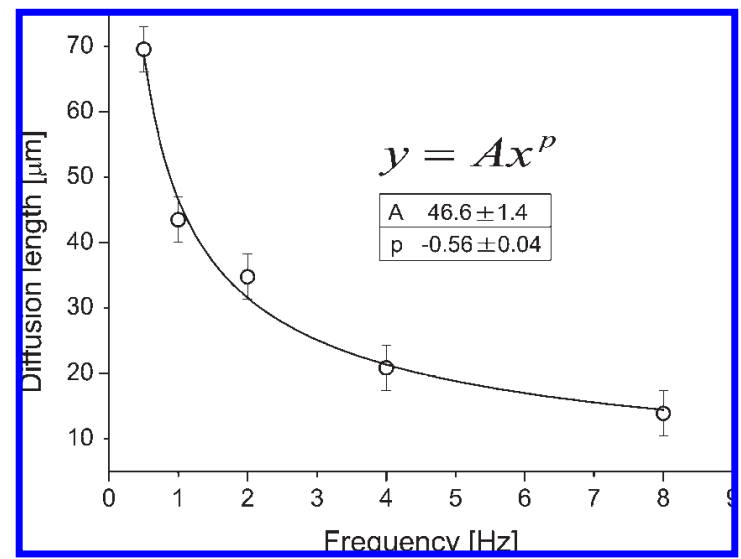

Figure 6. Diffusion length of Bodipy vs frequency. The amplitude of the signal was $6 \mathrm{~V}_{\mathrm{pp}}$. Data are fitted to a power-law model (continuous line).

Bodipy or Rhodamine. For instance, when dc signals were applied, $1 \mathrm{~V}$ was sufficient to observe changes in Fluorescein emission. On the other hand, $3 \mathrm{~V}$ were required to observe changes with the other dyes. Since the intensity of the emission of the Fluorescein is dependent on the $\mathrm{pH}$ of the solution, it is probable that electrolysis of water is responsible for the changes in fluorescence intensity. Water reactions lead to changes in the local $\mathrm{pH}$ value of the electrolyte.

Rhodamine $6 \mathrm{G}$ and Bodipy map concentrations of positive and negative ions, respectively. Since the two dyes were oscillating in phase, i.e., they both moved to the same electrode, the changes in the fluorescence emission of these two dyes are interpreted as an indication of the changes in the concentration of neutral salt (concentration polarization). This means that the electrolyte remains electroneutral but the ionic strength is higher where the fluorescence is brighter. Concentration polarization may appear as a consequence of Faradaic reactions ${ }^{37}$ and/or the nonlinear behavior of the diffuse layer. ${ }^{38,39} \mathrm{~K}^{+}$ and $\mathrm{Cl}^{-}$have almost equal diffusivities and, in this case, the frequency of the oscillation would be $2 \omega$ rather than $\omega$ for perfectly polarizable electrodes. ${ }^{38,39}$ Observations show that the ion concentration is oscillating with the same frequency as the field and, therefore, electrolysis of water would seem to be responsible for both changes in $\mathrm{pH}$ and changes in ionic concentration.

In summary, the experiments at low frequency in this simple geometry show that:

(1) the $\mathrm{pH}$ is lower near the positive electrode-the Fluorescein emission decreases there.

(2) the concentration of neutral salt is higher near the positive electrode-both Bodipy and Rhodamine $6 \mathrm{G}$ emissions increase there.

(38) Bazant, M. Z; Thornton, K.; Ajdari, A. Phys. Rev. E 2004, 70, 021506.

(39) Suh, Y. K.; Kang, S. Phys. Rev. E 2008, 77, 031504.
Depending on the solution $\mathrm{pH}$, electrolysis of water can occur under the two following scenarios: ${ }^{40}$

Acidic conditions

$$
\begin{aligned}
& 3 \mathrm{H}_{2} \mathrm{O} \rightarrow 2 \mathrm{H}_{3} \mathrm{O}^{+}+\frac{1}{2} \mathrm{O}_{2}+2 \mathrm{e}^{-} \quad \text { (anode) } \\
& 2 \mathrm{H}_{3} \mathrm{O}^{+}+2 \mathrm{e}^{-} \rightarrow 2 \mathrm{H}_{2} \mathrm{O}+\mathrm{H}_{2} \quad \text { (cathode) }
\end{aligned}
$$

while at basic conditions,

$$
\begin{aligned}
& 2 \mathrm{OH}^{-} \rightarrow \mathrm{H}_{2} \mathrm{O}+\frac{1}{2} \mathrm{O}_{2}+2 \mathrm{e}^{-} \quad \text { (anode) } \\
& 2 \mathrm{H}_{2} \mathrm{O}+2 \mathrm{e}^{-} \rightarrow 2 \mathrm{OH}^{-}+\mathrm{H}_{2} \quad \text { (cathode) }
\end{aligned}
$$

Electrochemical reactions in which ions are produced lead to an increase in salt concentration, while ion consumption leads to salt depletion. This situation is equivalent to the case of an electrical current flowing through semipermeable membranes. ${ }^{41}$ Experiments indicate that the total concentration of ions increases near the electrode where the electrolyte is acidified (anode). This leads to the conclusion that the reaction under acidic conditions is the dominant one- the $\mathrm{pH}$ decreases due to production of $\mathrm{H}_{3} \mathrm{O}^{+}$rather than consumption of $\mathrm{OH}^{-}$.

Typical frequencies for pumping fluids with microelectrode arrays are higher $\left(\sim 10^{2}-10^{3} \mathrm{~Hz}\right)$ than those used in this geometry. Nevertheless, these experiments provide an indication of how the charged dyes behave and, by inference, how electrode geometries other than the parallel plates used in this work might behave. Experiments were performed on a traveling-wave electrode array made of titanium microelectrodes $(20 \mu \mathrm{m}$ width and $20 \mu \mathrm{m}$ spacing) (see Figure 2). An array of the same characteristics was studied by Garcia-Sanchez et al., ${ }^{25}$ and it was found that for a $\mathrm{KCl}$ concentration of $10^{-4} \mathrm{M}$ the net fluid flow reversed at a threshold voltage of $4 \mathrm{~V}_{\mathrm{pp}}$.

For these experiments, a long microchannel was constructed with glass lid on top of the TW electrode array. The channel dimensions were $200 \mu \mathrm{m}$ high and $1 \mathrm{~mm}$ wide.This electrode/ electrolyte system allows observations from the side and from the top.The behavior was measured at low frequencies $(<10 \mathrm{~Hz})$, and we found the same behavior as for the two plates: Fluorescein becomes darker near the electrodes at positive potential, while Rhodamine $6 \mathrm{G}$ becomes brighter. To observe fluid motion, $500 \mathrm{~nm}$ diameter fluorescent beads were suspended in the fluid, and the electrode array was studied in the reverse pumping mode. When the TW signal was applied, it was found that the Fluorescein became darker. The fluorescence intensity decreased first near the electrodes, and after a few seconds, it was very low in the entire channel. This result indicates net production of $\mathrm{H}^{+}$with an applied ac signal, implying that Faradaic currents are not symmetric in time.

To verify that photobleaching was not responsible for this effect, observations were made without the electric field; photobleaching was only noticeable after several minutes. In contrast, the fluorescence diminished immediately after the electric field was applied. The reduction in light intensity indicates that the $\mathrm{pH}$ in the solution decreases. Figure 7 shows the fluorescence emission from the edge of the electrode array. Fluorescein dye was used, and a signal of $5 \mathrm{~V}_{\mathrm{pp}}$ and $500 \mathrm{~Hz}$ was applied. It can be seen that outside the array the fluorescence is unchanged but over the electrodes the electrolyte becomes dark. A sharp change in fluorescence intensity was observed between the electrolyte directly above the electrode array and the electrolyte outside this.

(40) Trau, M.; Saville, D. A.; Aksay, I. A. Langmuir 1997, 13, 6375-6381.

(41) Rubinstein, I.; Zaltzman, B. Phys. Rev. E 2000, 62, 2238-2251. 


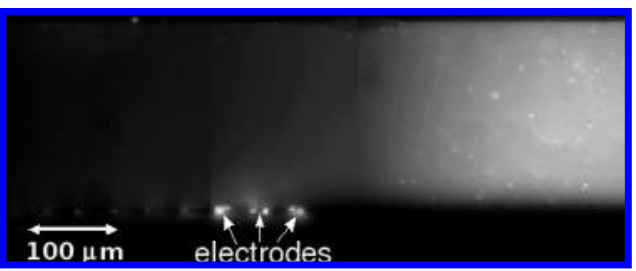

Figure 7. Fluorescein emission for a signal of $5 \mathrm{~V}_{\mathrm{pp}}$ at $500 \mathrm{~Hz}$ applied to a titanium TW array.The fluorescence decreases along the length of the array indicating a decrement in $\mathrm{pH}$.

Observations were also made at higher frequencies, which showed that the changes in fluorescence intensity are smaller than for $500 \mathrm{~Hz}$. In particular, for frequencies around $1 \mathrm{kHz}$, the fluorescein emission decreases, but higher voltages are also required $\left(7 \mathrm{~V}_{\mathrm{pp}}\right)$. For frequencies around $10 \mathrm{kHz}$, an obvious decrement in fluorescence was not seen even for a voltage amplitude of $20 \mathrm{~V}_{\mathrm{pp}}$ (maximum voltage provided by the generator). These observations do not mean that changes only occur for low frequencies. They mean that, for typical frequencies where flow reversal occurs, the changes in the liquid are considerable. Note that observation of a change in the fluorescein emission is only significant when the $\mathrm{pH}$ change is at least one unit; i.e., a change of 1 order of magnitude in the $\mathrm{H}^{+}$concentration.

The decrement of $\mathrm{pH}$ due to $\mathrm{H}_{3} \mathrm{O}^{+}$production is consistent with impedance measurements in previous work, ${ }^{26}$ where gradients in concentration of different ionic species were inferred. These gradients can play an important role in fluid flow generation.

2.2. Array Geometry and Channel Height Influence. In a previous paper, ${ }^{26}$ we reported a threshold voltage for reverse pumping for three different traveling-wave arrays. One device was made from titanium, with $20 \mu \mathrm{m}$ wide electrodes; the other two were platinum with 10 and $20 \mu \mathrm{m}$ wide electrodes. The threshold voltage was found to depend on both the size and the metal of the electrodes. The three electrode arrays were straight, and in each case, the height of the channel was $200 \mu \mathrm{m}$, much larger than the size of the electrodes. To investigate the influence of channel height, we fabricated two arrays made from platinum with $20 \mu \mathrm{m}$ wide electrodes. One electrode array was straight (consisting of 50 electrodes), and the other was circular (consisting of 400 electrodes).

For pumping experiments, the electrode array was covered with a channel made from polymer as shown in Figure 8 . The width of the channel was $450 \mu \mathrm{m}$, and the height was $50 \mu \mathrm{m}$. The channel formed a circular loop over the electrodes with inlet and outlet ports for loading. Two different input and output channels were used for injecting fluorescent beads used as tracer particles. The complete channel was filled with electrolyte, while the fluorescent beads were introduced into a small region for observation of flow. A similar method was described by Studer et al. ${ }^{15}$ For voltages below $3.5 \mathrm{~V}_{\mathrm{pp}}$ (the threshold previously reported for this $\operatorname{array}^{26}$ ) and frequencies of hundreds of hertz, it was observed that the fluid was pumped in the normal direction, as expected from previous results. At larger voltages, the fluid velocity was zero, even though flow reversal was expected. Bubbles appeared for a voltage amplitude of $8 \mathrm{~V}_{\mathrm{pp}}$. When the frequency was increased to $10 \mathrm{kHz}$, flow reversal was observed over an amplitude range of $6 \mathrm{~V}_{\mathrm{pp}}$ up to $16 \mathrm{~V}_{\mathrm{pp}}$ (the maximum we applied). In analogous experiments with asymmetric arrays of platinum electrodes, the channel height was $22 \mu \mathrm{m},{ }^{15}$ very close to the size of the wider electrode, and flow reversal was also found at higher frequencies than for normal flow. Flow reversal at higher frequencies for the asymmetric array may have a different physical origin than for the travelingwave system; nevertheless, we cannot discard the possible influence of the channel height for the asymmetric electrode structures. For example, we previously reported ${ }^{25}$ flow reversal with asymmetric arrays of titanium electrodes for increasing

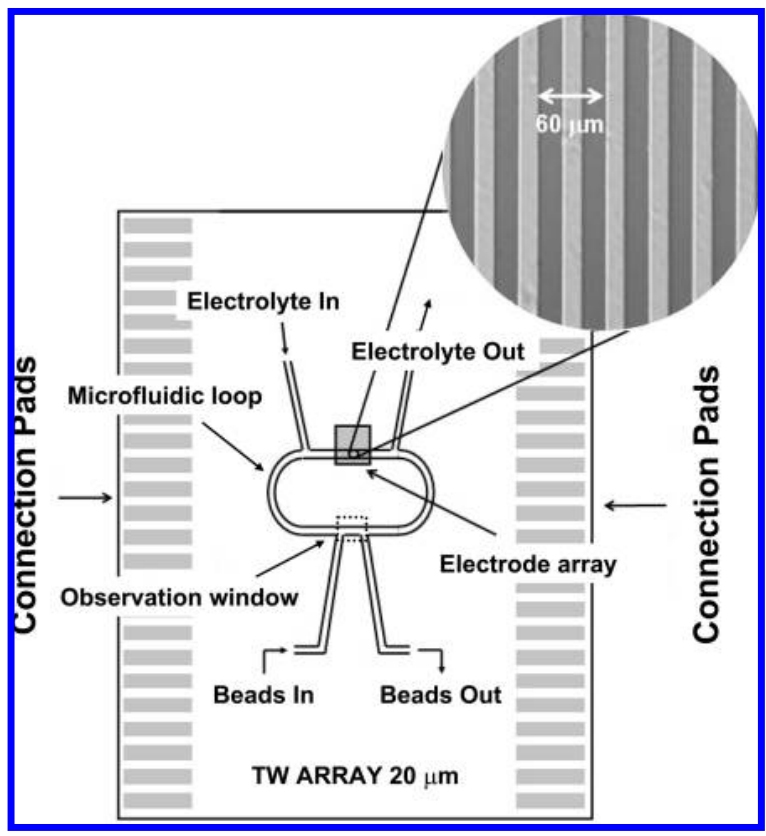

Figure 8. Microfluidic loop with a traveling-wave microelectrode array. Fluorescent beads are introduced into a small region for observation of flow. Each electrode in the array is connected to one of the connection pads. The four phase shifted ac signals are connected to the latter. A micrograph of the electrodes is included; the bright stripes there correspond to the electrodes.

voltage rather than frequency for a channel height larger than the electrode size $(260 \mu \mathrm{m}$ channel height against $100 \mu \mathrm{m}$ electrode width).

Figure 9 shows the experimental layout for the circular traveling-wave array. Two circular channels $1 \mathrm{~mm}$ wide were fabricated with PDMS (polydimethylsiloxane): one channel was $1 \mathrm{~mm}$ height and the other was $45 \mu \mathrm{m}$. They were placed on top of the circular traveling-wave array and filled with the electrolyte containing fluorescent beads. In the case of the tall channel $(1 \mathrm{~mm})$, the fluid behavior was as expected for normal pumping for an applied signal of hundreds of hertz and an amplitude below the threshold voltage $\left(3.5 \mathrm{~V}_{\mathrm{pp}}\right)$. Reverse pumping was observed at amplitudes above this threshold. In experiments with the shallow channel $(45 \mu \mathrm{m})$, normal pumping was observed at voltages below the threshold, but no net pumping was observed above. When higher frequencies were applied $(10 \mathrm{kHz})$, reverse pumping was clearly observed for a voltage amplitude of $10 \mathrm{~V}_{\mathrm{pp}}$. The results with the circular array are similar to those with the straight one.

End-effects due to the finite size of a TW array might have some influence on the fluid velocity; fresh liquid enters the array at one end. However, this effect is probably not significant, since for the circular array, flow reversal was observed for the same applied voltage and frequency as for the straight array. On the other hand, channel height was found to have a major influence on the flow reversal. Flow reversal was observed at higher frequencies and voltages for a channel height of $50 \mu \mathrm{m}$, much larger than the Debye length of the electrolyte $(\sim 30 \mathrm{~nm})$. Therefore, if the forces generating the motion are confined to the diffuse layer, a $50 \mu \mathrm{m}$ high channel should have no influence. This indicates that forces beyond the Debye length are responsible for flow reversal.

\section{Induced Charge beyond the Debye Length}

In this section, we discuss the origin of the electrical body forces and how these generate fluid motion over the traveling-wave microelectrode arrays. The goal is to describe situations where electrical charge is induced in the electrolyte bulk, creating a net 


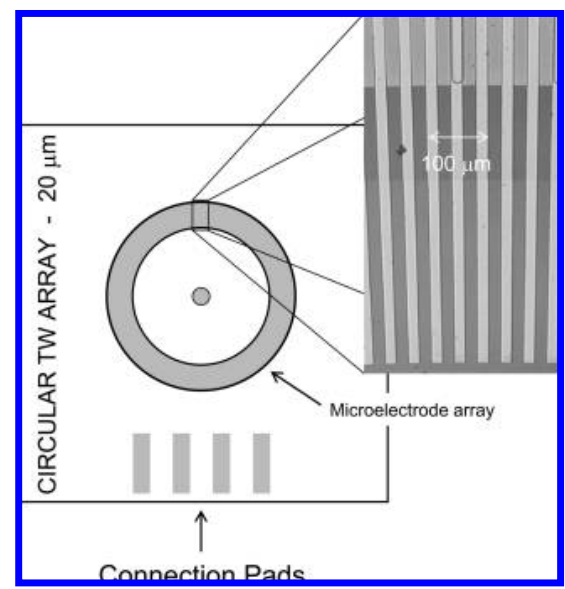

Figure 9. Circular traveling-wave microelectrode array. Each electrode in the array is connected to one of the connection pads. The four phase shifted ac signals are connected to the latter. A micrograph of the electrodes is included; the bright stripes there correspond to the electrodes.

fluid flow due to the action of the electric field on this charge. At low frequencies and low ionic concentrations, electrothermal effects can be discounted as the source of any appreciable motion. ${ }^{26}$ Therefore, we analyze only the case where conductivity gradients arise from electrochemical processes. Fluid velocity calculations are performed to evaluate the relative importance of the different terms in the body force and to obtain order of magnitude estimates for the velocity.

An electrical body force occurs when there is a net electrical charge in the liquid bulk. Electrolytes are usually quasi-electroneutral $\left(\rho / e \ll \Sigma_{i} c_{i}\right)$ at the micrometer scale. For instance, in the case of a 1:1 electrolyte, the relative difference in ion number densities corresponds to $\left(c_{+}-c_{-}\right) /\left(c_{+}+c_{-}\right)=\nabla \cdot(\varepsilon \mathbf{E}) / e\left(c_{+}+c_{-}\right)$ and is very small for saline solutions ${ }^{42,43} \sim \varepsilon \mathbf{E} / l e 2 c_{0} \ll 1$. An applied electric field can induce electrical charge when gradients in the concentration of ionic species are present, as shown below. The induced charge satisfies $\rho / e \ll \Sigma_{i} c_{i}$ although $\rho \mathbf{E}$ can be sufficient to generate observable fluid flow. Quasi-electroneutrality implies that the convection current can be neglected when compared to the ohmic current (see note ${ }^{44}$ ) and the electrical current in an electrolyte is written as ${ }^{37}$

$$
\mathbf{J}=-\sum_{i}\left[e\left|z_{i}\right| c_{i} \mu_{i}(\nabla \phi)+e z_{i} D_{i} \nabla c_{i}\right]
$$

where $\phi$ is the electric potential, $e$ the proton charge, and $z_{i}, D_{i}, \mu_{i}$, and $c_{i}$ are the ionic valence, diffusion coefficient, ionic mobility, and concentration of species $i$, respectively. From the continuity of the total electrical current, i.e., including the displacement current, $\nabla \cdot \mathbf{J}_{\text {tot }}=\nabla \cdot\left(\mathbf{J}-\varepsilon \partial_{\mathrm{t}} \nabla \phi\right)=0$ and the charge density $\rho$ in the bulk is given by

$$
\rho+\frac{\varepsilon}{\sigma} \frac{\partial \rho}{\partial t}=\frac{\varepsilon}{\sigma}\left(\nabla \sigma \cdot \nabla \phi+\sum_{i} e z_{i} D_{i} \nabla^{2} c_{i}\right)
$$

where $\sigma \equiv \Sigma_{\mathrm{i}} e\left|z_{i}\right| \mu_{\mathrm{i}} c_{\mathrm{i}}$ is the electrolyte conductivity.

(42) Saville, D. A. Annu. Rev. Fluid Mech. 1997, 29, 27-64

(43) Castellanos, A.; Ramos, A.; González, A.; Green, N. G.; Morgan, H. J. Phys. D: Appl. Phys. 2003, 36, 2584-2597.

(44) Negligible convection current is ensured by the assumption of quasielectroneutrality. Note that this does not imply that the flux of ions by convection can be neglected in general. The Péclet number may be important and, in that case, the mean concentration of ions could be affected by the fluid flow.
In a previous paper, ${ }^{26}$ we discussed situations in which the electric field acting on the induced charge could produce a nonzero time-averaged force $\langle\rho \mathbf{E}\rangle \neq 0$ and consequently net fluid flow. We explore these ideas further by numerically calculating the fluid velocity. In particular, we consider situations where the induced charge and the electric field are oscillating functions with the same frequency as the applied potential. If the driving field oscillates with frequency $\omega$, the induced charge density oscillates with $\omega$ for two cases: (a) if the conductivity gradient has a nonzero time average $\langle\nabla \sigma\rangle \neq 0$ and/or (b) the Laplacian of the concentration $\nabla^{2} c$ has a time-varying component with the same frequency as the electric field. Our experimental observations support these two possibilities: quasi steady-state gradients in conductivity due to nonsymmetric Faradaic reactions in ac signals and oscillations of the ionic concentration with the same frequency as the applied ac signal. In general, harmonics of higher order can appear in both the induced charge and the electric field when the applied voltage cannot be considered low, and components of $\rho \mathbf{E}$ can also contribute to a nonzero time-averaged force. We analyze the first terms in this expansion here.

3.1. Forces Due to Vertical Gradients in Conductivity. If the conductivity gradient has a quasi steady component, i.e., $\langle\nabla \sigma\rangle \neq 0$, the situation is similar to that of ac electrothermal flows described by Melcher et al. ${ }^{45}$ and Fuhr et al. ${ }^{46}$ - a wave of charge is induced in the bulk electrolyte. The characteristic frequency for this motion is the relaxation frequency of the liquid $(f=\sigma / 2 \pi \varepsilon)$. If the liquid conductivity is higher near the electrodes, a vertical gradient would lead to fluid flow in the direction of the reverse pumping mode.

At high voltages and frequencies, the electrochemical generation of conductivity gradients could be responsible for certain fluid flow observations, but at the frequencies used in this work, the influence of this mechanism is not clear. According to this mechanism, for $f \ll \sigma / 2 \pi \varepsilon$, the fluid velocity should increase with frequency, contrary to observation, although electrochemical reaction rates decrease with increasing frequency. In fact, the dependence of the conductivity gradients on the applied signal can be rather complicated. To evaluate the influence of this term, we assume that the nonsymmetrical electrochemical reactions generate a constant flux in conductivity (independent of frequency). The fluid velocity arising from this mechanism was calculated (for typical values of the flux). See Appendix A for the details.

Figure 10 shows the calculated net fluid velocity versus frequency, with parameters shown in Table 1. The conductivity is obtained from the diffusion equation in the steady state. The value of the conductivity gradient at the level of the electrodes is given by the imposed flux $-D(n \cdot \nabla \sigma)=\Gamma=2 \times 10^{-5} \sigma_{0}$ (S.I. units). This flux is on the order (or even higher) of that expected from experimental measurements. For this flux, the average conductivity in a $200 \mu \mathrm{m}$ height channel would be 10 times higher after $100 \mathrm{~s}$ (in agreement with previous observations ${ }^{26}$ ). As shown by the figure, the vertical gradient in conductivity can only generate noticeable fluid flow for frequencies above $10^{4} \mathrm{~Hz}$, larger than the typical frequencies reported for pumping. The electrode/electrolyte polarization was modeled as a surface impedance consisting of a resistor in parallel with a capacitor (see Appendix A). The resistance value (charge transfer resistance $R_{\mathrm{ct}}$ ) was parametrically changed within a wide range, but this had little influence on the results in Figure 10. This is because, at the frequencies where this mechanism is important,

(45) Melcher, J. R.; Firebaugh, M. S. Phys. Fluids 1967, 10, 1178-1185

(46) Fuhr, G.; Hagedorn, R.; Müller, T.; Benecke, W.; Wagner, B. J. Electromech. Svst. 1992, 1, 141-146. 


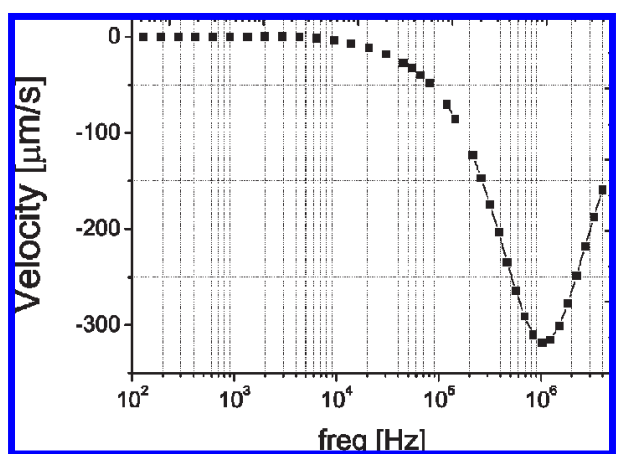

Figure 10. Net fluid velocity vs $\omega$ for a given constant flux on the electrodes $\left(-D(\mathrm{n} \cdot \nabla \sigma)=2 \times 10^{-5} \sigma_{0}\right) . V_{0}=2 \mathrm{~V}$. Fluid velocity is important for frequencies above $10^{4} \mathrm{~Hz}$.

Table 1. Parameters for the Fluid Velocity Computations

$\begin{array}{ll}\Gamma / \sigma_{0} & 2 \times 10^{-5} \mathrm{~m} / \mathrm{s} \\ \sigma_{0} & 1.5 \mathrm{mS} / \mathrm{m} \\ \mathrm{L} & 160 \mu \mathrm{m} \\ \mathrm{D} & 2 \times 10^{-9} \mathrm{~m}^{2} / \mathrm{s} \\ V_{0} & 2 \mathrm{~V} \\ \eta & 10^{-3} \mathrm{~Pa} \cdot \mathrm{s}\end{array}$

the capacitive element of the impedance is so low that $R_{\mathrm{ct}}$ has little influence. At frequencies around the charge relaxation frequency, there is a phase lag between the field and the induced charge, so that the charge experiences a force and the liquid moves along the electrode array. At low frequencies, the induced charge has sufficient time to follow the field vector without time delay, and the time-averaged horizontal force is zero. In the computations, the conductivity near the electrodes is $\sigma \simeq 3 \sigma_{0}=4.5 \mathrm{mS} / \mathrm{m}$. The corresponding relaxation frequency for this conductivity is $f=960 \mathrm{kHz}$, indicating why in Figure 10, the maximum velocity is around $1 \mathrm{MHz}$. The observation of flow reversal at $10 \mathrm{~V}_{\mathrm{pp}}$ and $10 \mathrm{kHz}$ for low channel heights may be related to this mechanism.

3.2. Forces Due to Longitudinal Conductivity Gradients. The typical frequencies required to generate net flow in the presence of a vertical conductivity gradient are high because a phase lag between the field and the induced charge is required. Nevertheless, the case of a spatial phase difference between an oscillating induced charge $\rho(\omega)$ and the electric field $\mathbf{E}(\omega)$ has not been discussed. Consider the situation shown in Figure 11, where the conductivity changes periodically along the electrode array but is shifted a certain distance $(L \varphi / 8 \pi)$ with respect to the "unit cell" of an electrode. The physical origin of such gradients in conductivity could be twofold: first, the electrical current through the electrodes may be strongly nonhomogeneous (contrary to the hypothesis in the previous section where flux was assumed constant along the electrode). Second, if fluid velocities are important, the convection of $\sigma$ could generate spatial variations in conductivity. Therefore, for the sake of completeness, it is interesting to evaluate the influence of longitudinal gradients of conductivity on fluid flow. Both the conductivity and the spatial phase difference can depend on the applied signal in a complicated fashion. To analyze this mechanism, assume that $\sigma$ at the level of the electrodes is given by the expression in Figure 11 and satisfies the diffusion equation in the bulk. This corresponds to a conductivity that varies smoothly along the electrode array, with a minimum value equal to $\sigma_{0} . \Delta \sigma$ is chosen to be small enough $\left(\Delta \sigma \ll \sigma_{0}\right)$ so that the conductivity gradients are moderate. Note that the spatial period is chosen to be $T=L / 4$; each electrode is equivalent, and therefore, we

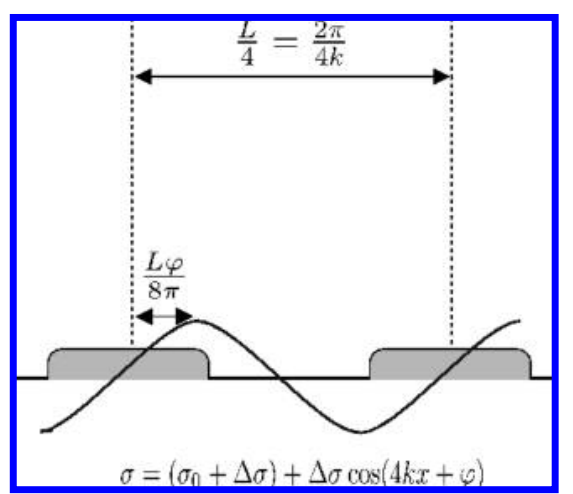

Figure 11. The conductivity at the level of the electrodes is supposed to change periodically along the array in order to evaluate the effect of longitudinal conductivity gradients.

expect changes in conductivity to have the same spatial period. The term $(\varepsilon / \sigma) \partial \rho / \partial t$ in expression 6 is negligible for the typical frequencies used in the experiments and can be neglected. In this way, the effect of a spatial phase difference can be analyzed separately from that of a phase lag.

The problem studied in this section has some similarities to the work of Trau et al. ${ }^{40}$ They considered the concentration polarization over an electrode generated by an electrical current. Electrical charge is induced within the polarization layer, and when this layer is perturbed by the presence of colloidal particles, lateral flow is generated in the system leading to assembly of the colloidal particles. This perturbation was modeled as a spatial sinusoidal perturbation of the electrical current, and in this sense, it is similar to the sinusoidal variation of conductivity that is proposed here. A difference is that, in the work of Trau et al., they study the case of a dc field, arguing that in ac a nonzero average force would also appear, although they did not explicitly consider that case. Here, we consider the case of ac fields acting on gradients of conductivity generated by Faradaic currents. A similar problem was also considered by Ristenpart et al. ${ }^{47}$ They studied the electroosmotic flow generated over electrodes with nonuniform Faradaic currents. This situation could have some similarities with the problem in this section if the gradients of conductivity were generated by nonhomogeneous currents. There is, however, an important difference. In the work by Ristenpart et al., the flow is generated by the electric field acting on the diffuse charge of the electrical double layer. In our case, we consider a quasielectroneutral liquid and the force appears as a consequence of the electric field acting on horizontal gradients of conductivity in the bulk.

Figure 12 shows the calculated net velocity versus the spatial phase difference $\varphi$ for $\Delta \sigma / \sigma_{0}=0.1, V_{0}=2 \mathrm{~V}$, the typical frequency for ACEO, $\omega=k \sigma \lambda_{\mathrm{D}} / \varepsilon=2510 \mathrm{rad} / \mathrm{s}$ and for two different values of $R^{\prime}=\sigma_{0} k R_{\mathrm{ct}}$. Depending on the sign of the spatial phase difference, a net fluid velocity is obtained in one direction or the other. The case of $\varphi<0$ corresponds to that depicted in Figure 11. This conductivity profile would generate fluid motion in the same direction as the potential wave moves. On the other hand, if $\varphi>0$, net fluid motion is generated in the opposite direction (reverse flow). Maximum velocities are found for a phase difference of $\pm \pi / 2$. The fluid on an electrode flows toward the side with higher conductivity.

Longitudinal gradients of conductivity could be generated by nonuniform Faradaic reactions on the electrodes and/or by ionic

(47) Ristenpart, W. D.; Jiang, P.; Slowik, M. A.; Punckt, C.; Saville, D. A.; Aksay, I. A. Langmuir 2008, 24, 12172-12180. 


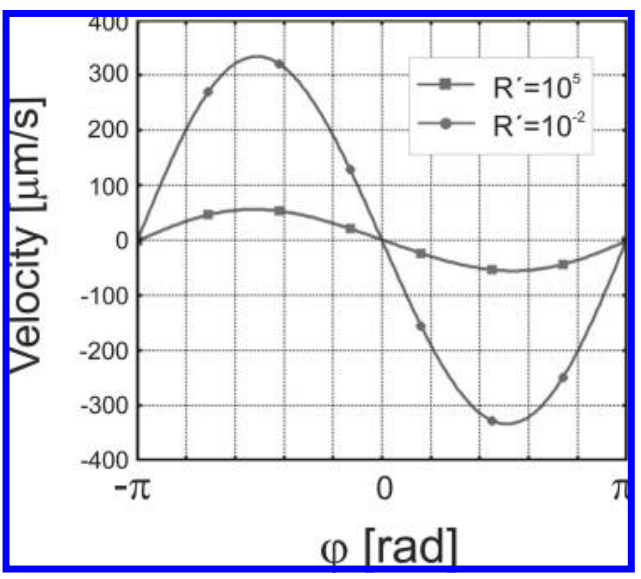

Figure 12. Net fluid velocity due to a longitudinal conductivity gradient like that in Figure 11. The velocity is plotted vs spatial phase difference $\varphi .\left(\omega=k \sigma \lambda_{\mathrm{D}} / \varepsilon, \Delta \sigma / \sigma_{0}=0.1\right.$, and $\left.V_{0}=2 \mathrm{~V}\right)$.

concentration convection, although these mechanisms are not clear at present.

3.3. Induced Charge Due to Different Ionic Mobilities. The term $\Sigma_{i} e z_{i} D_{i} \nabla^{2} c_{i}$ in eq 6 could yield net free charge $\rho=$ $(\varepsilon / \sigma) \sum_{i} e z_{i} D_{i} \nabla^{2} c_{i}$ oscillating at $\omega$. For a symmetric binary electrolyte, under the quasi-electroneutrality approximation $c_{+}=c_{-}=c, c$ satisfies the diffusion equation $\partial_{t} c=D_{\mathrm{a}} \nabla^{2} c$, where $D_{\mathrm{a}}$ is the ambipolar diffusion coefficient $\left(D_{\mathrm{a}}=2\left(D_{+} D_{-}\right) /\right.$ $\left.\left(D_{+}+D_{-}\right)\right)$. Electrical charge is induced in a layer of typical thickness $\delta=\left(D_{\mathrm{a}} / \omega\right)^{1 / 2}$, i.e., the diffusion layer. For low voltages, $c$ can be written as $c=c_{0}+\operatorname{Re}[\tilde{c} \exp i \omega t]$ with $\tilde{c}$ a solution of

$$
D_{a} \nabla^{2} \tilde{\mathrm{c}}=i \omega \tilde{\mathrm{c}}
$$

The typical length for $\tilde{c}$ is $\delta$, and for typical values of $D_{\mathrm{a}} \sim 10^{-9} \mathrm{~m}^{2} / \mathrm{s}$ and $\omega \sim 10^{3} \mathrm{rad} / \mathrm{s}$, the thickness of this layer is on the order of micrometers, $\delta \sim 1 \mu \mathrm{m}$. Note that, for a binary symmetrical electrolyte with equal diffusivities $D_{+}=D_{-}$ (e.g., $\mathrm{KCl}$ ), the induced charge is identically zero.

This force has been considered ${ }^{48,49}$ when describing the motion of a spherical particle on top of ac polarized electrodes. A Faradaic current was assumed to flow through the electrodes. In our experiments, fluorescence intensity oscillations at low frequencies (section 2.1) showed that the ionic concentration oscillates with the same frequency as the applied voltage. $\mathrm{Cl}^{-}$is the negative ion that provides electroneutrality when the concentration of $\mathrm{H}^{+}$increases. Since the mobility difference between the two is large $\mu_{\mathrm{H}+} / \mu_{\mathrm{Cl}-}=D_{\mathrm{H}+} / D_{\mathrm{Cl}-}=4.6$, a considerable induced charge is to be expected. For simplicity, the calculations assume a binary electrolyte with this mobility ratio. This approximation is reasonable if the concentration of $\mathrm{H}^{+}$ overrides the $\mathrm{K}^{+}$concentration near the electrodes. Furthermore, for $\left[\mathrm{H}^{+}\right] \gg\left[\mathrm{OH}^{-}\right]$the recombination of these two ions can be neglected against the diffusion within distances of a few micrometers $\left(k_{\mathrm{r}} c_{+} c_{-} \ll D_{+} \nabla^{2} c_{+}\right.$, where $k_{\mathrm{r}}$ is the constant for recombination). In effect, $c_{-}$can be expected to be $c_{-} \leq 10^{-9} \mathrm{~mol} / \mathrm{L}$ from the experiments, and taking $k_{\mathrm{r}}=1.4 \times 10^{-11} \mathrm{~L} / \mathrm{mol} \cdot \mathrm{s}($ ref 50) the recombination term can be neglected for distances $l \ll 10 \mu \mathrm{m})$.

For simplicity, we calculate the fluid velocity generated by this mechanism for low applied voltages. Under this approximation, the concentration of ions $c$ does not change much $\left(\left|c-c_{0}\right| / c_{0}\right) \ll$ 1 ( $c_{0}$ is the bulk concentration), and consequently, the induced

(48) Sides, P. J. Langmuir 2001, 17, 5791-5800.

(49) Fagan, J. A.; Sides, P. J.; Prieve, D. C. Langmuir 2004, 20, 4823-4834.

(50) Eigen, M.; de Maeyer, L. Proc. R. Soc. London, Ser. A 1958, 247, 505-533. charge is expected to oscillate at frequency $\omega$, that is, $\nabla^{2} \tilde{c}(\omega)$ $c(\omega) \simeq \nabla^{2} \tilde{c}(\omega) / c_{0}$. Therefore, the time-averaged electrical body force is written as

$$
\langle\mathbf{f}(t)\rangle=-\frac{1}{2} \frac{\varepsilon k_{\mathrm{B}} T}{e} \gamma \operatorname{Re}\left[\frac{\nabla^{2} \tilde{\mathrm{c}}(\omega)}{c_{0}} \cdot(\nabla \Phi)^{*}\right]
$$

where $\gamma=\left(D_{+}-D_{-}\right) /\left(D_{+}+D_{-}\right)$and the fluid velocity scales with the square of the applied voltage amplitude $V_{0}$. We have considered two ionic species $\left(\mathrm{H}^{+}\right.$and $\left.\mathrm{Cl}^{-}\right)$. The ionic concentration $\tilde{c}$ is a solution of eq 7 with boundary conditions on the electrodes ${ }^{37,51}$

$$
\mathbf{n} \cdot \nabla \tilde{\mathbf{c}}=\frac{J_{\mathrm{F}}}{2 e D_{\mathrm{H}^{+}}}
$$

where the Faradaic current $J_{\mathrm{F}}$ is due to electrochemical reactions of $\mathrm{H}^{+}$. This boundary condition is correct if the displacement current is negligible compared with the Faradaic current, i.e., the flux of species in the diffusion layer is driven mainly by the Faradaic current (see Appendix B). This is a justified simplification for the present case, because it is to be expected that Faradaic currents depolarize the interface. The Faradaic current is written as $J_{\mathrm{F}}=\Delta \Phi / R_{\mathrm{ct}}$, where $\Delta \Phi$ is the voltage drop through the EDL and $R_{\mathrm{ct}}$ is the resistance of the resistor in the Randles impedance (Appendix A). The electric potential is a solution of Poisson's equation written as

$$
\nabla^{2} \Phi=-\frac{k_{\mathrm{B}} T}{e} \gamma \frac{\nabla^{2} \tilde{\mathrm{c}}(\omega)}{c_{0}}
$$

Solving the Stokes equation with expression 8 as body force gives the velocity generated by this mechanism. Figure 13 shows the net fluid velocity (scaled by the signal amplitude squared $V_{0}^{2}$ ) versus the frequency of the applied signal for two values of $R^{\prime}=R_{\mathrm{ct}} \sigma_{0} k$. The other parameters in calculations are listed in Table 1 (i.e., the parameters of the experiments described in a previous paper ${ }^{26}$ ). The net velocity corresponds to reverse flow (a direction opposite to that of the TW). The calculations show that, when the more mobile ion undergoes chemical reactions, flow reversal is expected at typical frequencies of experiments.

In these calculations, $D_{+} / D_{-}=4.6$, i.e., $\gamma=0.64$. If $\gamma<0$, this mechanism generates flow in the same direction as the traveling-wave. Since the $\mathrm{H}^{+}$ion is more mobile than $\mathrm{Cl}^{-}(\gamma>0)$, this mechanism provides a good explanation for the flow reversal. In ref 52, preliminary analytical results were reported for the solution of the full linear electrokinetic equations considering a single mode traveling-wave and the effect of Faradaic charging and asymmetric mobilities. These analytical results are in agreement with the calculations presented here.

It is interesting to note that the frequency dependence of the net fluid velocity for the flow reversal reported in experiments ${ }^{26}$ is in good agreement with the calculations for $R^{\prime}=1$ in Figure 13. The impedance measurements in that paper showed a strong decrement at low frequencies after application of signals that lead to flow reversal. The surface impedance became the same as the bulk resistance, that is, $R_{\mathrm{ct}} \sim 1 / \sigma k$. Therefore, we consider $R^{\prime}=1$ a reasonable value. Setting the

(51) Sides, P. J. Langmuir 2003, 19, 2745-2751.

(52) González, A.; Ramos, A.; García-Sánchez, P.; Castellanos, A. arXiv/ $0803.1055,2008$ 


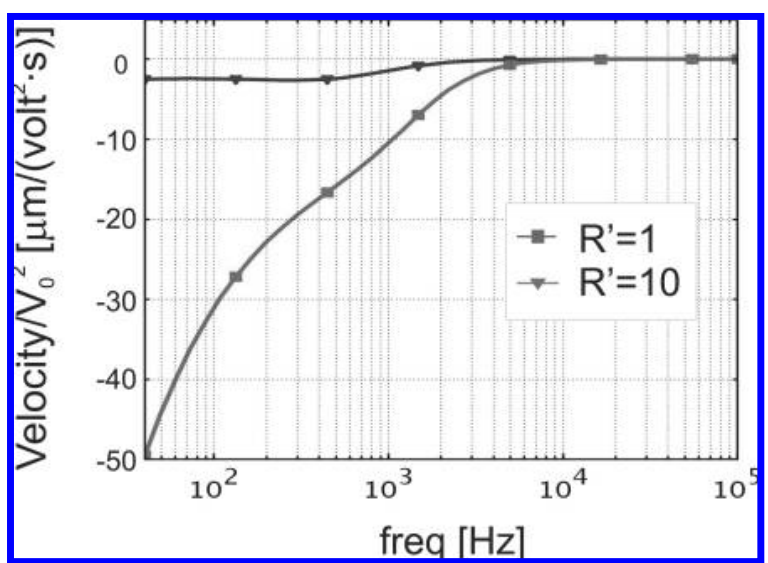

Figure 13. Net fluid velocity normalized by square of the voltage amplitude plotted against frequency for induced charge due to two different ionic mobilities. $D_{+} / D_{-}=4$.6, i.e., $\gamma=0.64$.

signal amplitude $V_{0}$ to that typical of flow reversal $\left(3.5-8 \mathrm{~V}_{\mathrm{pp}}\right)$, the experimental velocity values are smaller than the computed velocity values for $R^{\prime}=1$. For example, for a signal with 3.5 $\mathrm{V}_{\mathrm{pp}}$ at $300 \mathrm{~Hz}$ the experimental net velocity in ref 26 is $42 \mu \mathrm{m} / \mathrm{s}$, while for $4.5 \mathrm{~V}_{\mathrm{pp}}$ and $400 \mathrm{~Hz}$, the velocity is $69 \mu \mathrm{m} / \mathrm{s}$. For 3.5 $\mathrm{V}_{\mathrm{pp}}$ and $300 \mathrm{~Hz}$, the computations for $R^{\prime}=1$ predict a velocity of $61 \mu \mathrm{m} / \mathrm{s}$, and for $4.5 \mathrm{~V}_{\mathrm{pp}}$ and $400 \mathrm{~Hz}$, the corresponding velocity is $86 \mu \mathrm{m} / \mathrm{s}$. In general, experimental velocities are systematically lower than the computations for $R^{\prime}=1$ (around 30\%). This is satisfactory since we do not account for traveling-wave electroosmosis where net motion is generated in the other direction.

This mechanism is probably responsible for the flow reversal observed with electrode arrays driven by TW potentials. However, it is important to be aware that, for the typical signal amplitudes used in experiments, the approximation of low voltages is not true, and unrealistic values for $\left|c-c_{0}\right|$ would be obtained with the linear equations (electrical current would become greater than the diffusion limited current). Note that this is a common feature with the linear and weakly nonlinear theories of ACEO (even in the case of perfectly polarizable electrodes). At typical voltages of the experiments, the expected values of the variations in ion concentration become so high that the perturbation analysis results are inconsistent. Although the Faradaic current is effectively limited, the term $\nabla^{2} c / c$ is not bounded, since $c$ can approach zero. As the voltage increases, oscillations of $c$ are greater, and consequently, the induced charge and the force due to the difference in ionic mobilities can be very high. Finally, it is worth considering the work of Gregersen et al. ${ }^{17}$ where flow reversal was observed in platinum asymmetric arrays at low voltage and frequency for higher concentrations than we use here $(400 \mu \mathrm{M})$. That "unexpected" reversal could be related to the mechanism discussed here, since the electroosmotic motion decreases as concentration increases.

\section{Conclusions}

Experimental work has been performed to evaluate the influence of different factors on the pumping of electrolytes with microelectrode arrays subjected to traveling-wave potentials. The same behavior was found for a linear array and a circular one. The height of the microfluidic channel influenced pumping performance. Flow reversal requires higher voltages and frequencies for shallow channels. Experiments with fluorescent dyes indicated that electrochemical reactions occur at the electrodes, leading to a $\mathrm{pH}$ decrement as well as concentration polarization that oscillates with the frequency of the applied signal. These results lead to the conclusion that pumping mechanisms where the electrical forces are located beyond the Debye length can be important. In a previous paper, ${ }^{26}$ we discussed how induced charge due to Faradaic currents could appear in the electrolyte bulk and generate net flow. We have now modeled this effect for the TW array and calculated fluid velocities for example cases in order to discuss the possible influence of different mechanisms. When a vertical conductivity gradient is present, the generated velocity for typical signals is negligible at frequencies much smaller than the relaxation frequency of the electrolyte (corresponding to the typical frequencies of the reported motion) but could be important above $10 \mathrm{kHz}$. Observations of flow reversal in shallow channels may be related to this. Longitudinal conductivity gradients could give rise to large net velocities, although the mechanisms generating these gradients are not clear at present. Finally, the induced charge due to the difference in mobilities between ionic species seems to be important and qualitatively explains the flow reversal previously reported. Since electrochemical reactions at the electrodes are central to the proposed mechanisms, it would be desirable to use alternative electrode materials and examine how these might influence the experimental observations reported here. For example, in ref 26, the threshold voltage for flow reversal was shown to depend on the material of the electrode array. Further theoretical studies are required in order to analyze this mechanism for different voltage amplitudes and concentrations, i.e., an analysis beyond the linear approximation.

Acknowledgment. We acknowledge the financial support of the Spanish government agency DGCyT and Junta de Andalucía under contracts FIS2006-03645 and FQM-241, respectively. PGS acknowledges to Stuart Williams his assistance in the fabrication of the shallow PDMS channel for the circular TW array.

\section{Appendix A}

In this appendix, we summarize the equations and boundary conditions in the computations of sections 3.1 and 3.2.

(I) The electric field is given by the charge conservation equation $\nabla \cdot((\sigma+i \omega \varepsilon) \mathbf{E}=0)$. The polarization of the electrode/electrolyte interface is modeled by a Randles type surface impedance in the absence of a Warburg impedance $Z=R_{\mathrm{ct}} /\left(1+i \omega C R_{\mathrm{ct}}\right)$ (corresponding to a resistor and a capacitor in parallel), $C$ is the capacitance (per unit area) of the interface, and $R_{\mathrm{ct}}$ is the specific charge transfer resistance, i.e., the area-specific resistance to Faradaic currents. ${ }^{27,37}$ For simplicity, the Warburg impedance is not considered; it could have an important role for low $R_{\mathrm{ct}}$ and low frequencies. ${ }^{28}$ The double layer capacitance $C$ is estimated from the DebyeHuckel theory as $C=\varepsilon / \lambda_{\mathrm{D}}{ }^{53}\left(\lambda_{\mathrm{D}}\right.$ is the Debye length, related to the diffusion coefficient by $\left.\lambda_{\mathrm{D}}=(D \varepsilon / \sigma)^{1 / 2}\right)$. The boundary condition on the electrodes is then

$$
-\mathbf{n} \cdot \nabla \Phi=\frac{V-\Phi}{\sigma Z}
$$

(53) Hunter, R. Introduction to Modern Colloid Science; Oxford University Press, 1993 
where $V$ is the applied potential and $\Phi$ is the electric potential phasor $(\phi=\operatorname{Re}[\Phi \exp (i \omega \mathrm{t})])$. On the gap between electrodes, the boundary condition corresponds to zero normal current, i.e., $\mathbf{n} \cdot \nabla \Phi=0$.

(II) The conductivity is obtained by solving the diffusion equation that reduces to Laplace equation:

$$
\nabla^{2} \sigma=0
$$

At the top of the domain, there is a constant conductivity $\sigma=\sigma_{0}$. At the interelectrode gap, the condition is that of zero flux, $\mathbf{n} \cdot \nabla \sigma=0$. The conductivity gradient on the electrodes is fixed to a constant value, $-D(\mathbf{n} \cdot \nabla \sigma)=\Gamma$, the value of this constant then determines a net flux.

(III) The fluid velocity is obtained from the Stokes equations with electrical body forces:

$$
\begin{gathered}
\nabla \cdot \mathbf{u}=0 \\
-\nabla p+\eta \nabla^{2} \mathbf{u}=-\langle\rho \mathbf{E}\rangle
\end{gathered}
$$

where $\mathbf{u}=u \mathbf{e}_{x}+v \mathbf{e}_{z}$ is the velocity field and $p$ the pressure. In this case, $\tilde{\rho}=\varepsilon(\nabla \tilde{\sigma} / \tilde{\sigma}) \cdot \nabla \Phi$ with $\tilde{\sigma}=\sigma+i \omega \varepsilon$. The timeaveraged force is then written as $\langle\tilde{\rho} \mathbf{E}\rangle=(1 / 2) \operatorname{Re}\left[\tilde{\rho} \mathbf{E}^{*}\right]$.

No slip is imposed at the electrodes and at the interelectrode gaps. Note that no electroosmotic flow is considered here despite the polarization of the electrodes, accounting for the computation of the electric potential. We only analyze the contribution of the induced charge in the electrolyte bulk. At the top of the domain, the stress tensor is set to zero (neutral boundary condition). The velocity is homogeneous there $\left(\mathbf{u}=u \mathbf{e}_{x}\right.$ with $\left.\partial u / \partial z=0\right)$ and corresponds to the net pumping velocity generated by the array.

\section{Appendix B}

In this appendix, we demonstrate that boundary condition 9 is correct in the limit when the displacement current is negligible compared with the Faradaic current. The ionic species fluxes are

$$
F_{+}=-D_{+} \frac{\partial c_{+}}{\partial z}-\mu_{+} c_{+} \frac{\partial \phi}{\partial z}
$$

$$
F_{-}=-D_{-} \frac{\partial c_{-}}{\partial z}+\mu_{-} c_{-} \frac{\partial \phi}{\partial z}
$$

and the 1-D conservation equations of the species are written

$$
\begin{gathered}
\frac{\partial c_{+}}{\partial t}=-\frac{\partial F_{+}}{\partial z} \\
\frac{\partial c_{-}}{\partial t}=-\frac{\partial F_{-}}{\partial z}
\end{gathered}
$$

dividing eq 17 by $D_{+}$and eq 18 by $D_{-}$, adding both results and integrating from the electrode $(z=0)$ to the end of the diffuse layer $\left(l \gg \lambda_{\mathrm{D}}\right)$, we obtain

$$
\begin{aligned}
& \int_{0}^{l}\left(\frac{1}{D_{+}} \frac{\partial c_{+}}{\partial t}+\frac{1}{D_{-}} \frac{\partial c_{-}}{\partial t}\right) \mathrm{d} z \\
& \quad=\left(\frac{F_{+}}{D_{+}}+\frac{F_{-}}{D_{-}}\right)_{z=0}-\left(\frac{F_{+}}{D_{+}}+\frac{F_{-}}{D_{-}}\right)_{z=l}
\end{aligned}
$$

If the cation is the reactive ion, at the electrodes $F_{+}=J_{\mathrm{F}} / e$, $F_{-}=0$, and

$$
\left(\frac{F_{+}}{D_{+}}+\frac{F_{-}}{D_{-}}\right)_{z=0}=\frac{J_{\mathrm{F}}}{e D_{+}}
$$

Outside the Debye layer $l>\lambda_{\mathrm{D}}, c_{+}=c_{-}=c$, and

$$
\left(\frac{F_{+}}{D_{+}}+\frac{F_{-}}{D_{-}}\right)_{z=l}=-2 \frac{\partial c}{\partial z}
$$

Introducing $\rho / e=c_{+}-c_{-}$and $c=\left(c_{+}+c_{-}\right) / 2$, the left-hand side of eq 19 is

$$
\int_{0}^{l}\left(\frac{1}{D_{+}}+\frac{1}{D_{-}}\right)\left(\frac{\partial c}{\partial t}-\gamma \frac{\partial \rho / 2 e}{\partial t}\right) \mathrm{d} z
$$

The first term is always smaller than $\partial c / \partial z$ because it is on the order of $\omega c \lambda_{\mathrm{D}} / D_{a}$ and $\partial c / \partial z$ is on the order of $c / \delta$. The second term is on the order of $q \omega / e D_{+}$, where $q$ is the surface charge in the diffuse layer. For the case where $q \omega / e D_{+} \ll \partial c / \partial z$, the boundary condition 9 is correct, $(20)=(21)$, i.e. when the displacement current $\omega q \ll e D_{+} \partial c / \partial z=J_{\mathrm{F}} / 2$. 